\title{
Jejunal Obstruction
}

National Cancer Institute

\section{Source}

National Cancer Institute. Lejunal Obstruction. NCI Thesaurus. Code C78398.

Blockage of the normal flow of the small intestinal contents in the jejunum. 